\title{
Complete Hydrogen Storage System by ISRU
}

\author{
Peter J. Schubert, Ph.D., P.E., Senior Member AIAA \\ IUPUI, Indianapolis, IN 46202
}

\begin{abstract}
New technologies make it possible to build in space a complete hydrogen storage system using ISRU methods and techniques. Hydrogen can be stored in a solid-state form on the surface atoms of high surface area matrices such as those of porous silicon. Silicon is abundant in regolith and can be purified using a purely mechanical means which results in particulates in the scale range of tens of nanometers. Reagents used to porosify these nano-particles can be regenerated thermally to essentially eliminate the need for resupply from earth. Catalysts are needed to divide dihydrogen gas into atomic hydrogen for solid-state adsorption and to mediate the temperatures and pressures of charge and discharge into ranges easily achievable with simple equipment. Recent research has identified the utility of non-platinum group catalyst materials which are widespread on the moon. Rapid discharge, needed for propulsion, is possible with infra-red illumination at wavelengths which pass through pure silicon but are absorbed by the silicon-hydrogen bond. Such IR emitters can be fabricated by embossing of silica and additive manufacturing of metals. Control and power electronics can be fabricated using a patented process designed for space operations, and built on either silicon or silicon carbide substrates derived from regolith. Bringing these five technologies together for the first time allows a system which can be fed with moderate pressure gaseous hydrogen at moderate temperatures, stored for long durations with minimum loss, then released upon demand across a wide range of controllable rates. Such a system can displace the need for cryogenic hydrogen storage. Being suitable to bottom-up fabrication using only in-space materials makes this a "green" ISRU technology to store hydrogen for fuel cells, rocket engines, and chemical processes.
\end{abstract}

\section{Introduction}

$\mathrm{H}$ ydrogen in space is abundant, and important. When combined with oxygen, hydrogen provides on-demand electricity, water, and propulsion. Solid-state storage of hydrogen has many advantages over cryogenic (liquid) hydrogen storage. The system presented herein for the first time has the further advantage of being entirely made by in situ resource utilization (ISRU), using regolith, sunlight, and vacuum. Five novel technologies are introduced which, taken together, provide an affordable and convenient hydrogen storage system for use in space.

Cryogenic storage of hydrogen has enormous technical challenges. It must be cooled to near absolute zero and kept away from any source of heat, which is impossible. Evaporated hydrogen expands rapidly, thus venting is needed to prevent the tank from exploding. Metals exposed to the extreme cold of liquid hydrogen become brittle, and minute cracks in welded seams can allow leakage. Transfer of hydrogen is difficult, and movement of liquid is a challenge in microgravity environments. Many space agencies eye the hydrogen trapped in permanently-shadowed lunar craters as a valuable resource. Extracting and then storing this valuable element has great import for future space endeavors. Figure 1 shows a schematic flow of activities to produce hydrogen for use, and for sale, from lunar regolith.

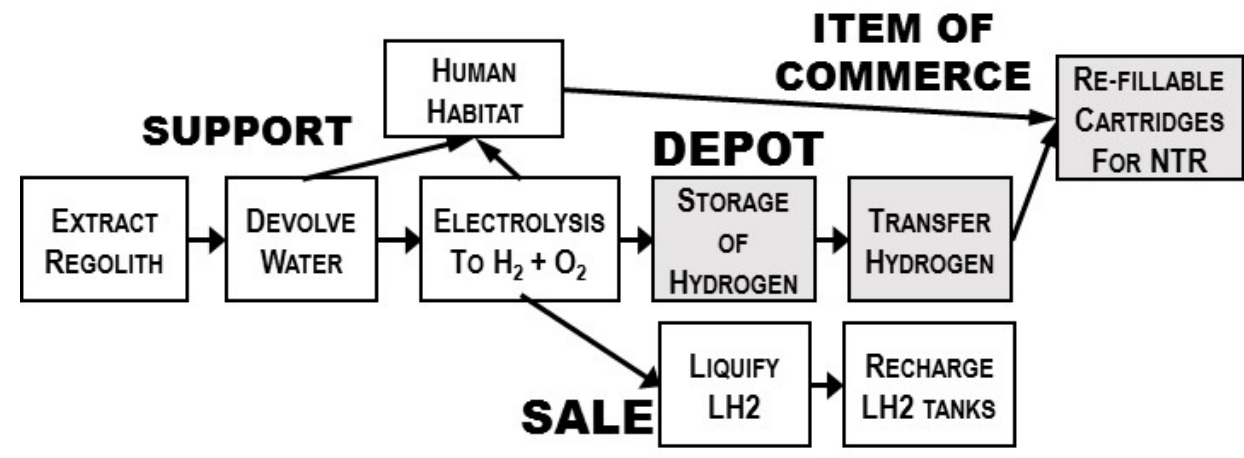

Figure 1. Hydrogen extraction and storage. Shaded boxes addressed herein.

This is the author's manuscript of the article published in final edited form as:

Schubert, P. J. (2018). Complete Hydrogen Storage System by ISRU. In 2018 AIAA SPACE and Astronautics Forum and Exposition. 


\section{Solid-State Hydrogen Storage}

R recent US patents have been issued for solid-state hydrogen storage using catalytically-modified porous R.silicon ${ }^{1,2,3,4}$, and a Phase I STTR feasibility study, funded by the National Science Foundation ${ }^{5}$, was recently completed. The key innovation is to use the high specific surface area silicon which has been made porous by electrochemical etching. Pores of 3.5 nanometer diameter and up to 250 microns deep yield $800 \mathrm{~m}^{2} / \mathrm{gm}$ of surface silicon dangling bonds capable of storing atomic hydrogen. Small clusters of metal atoms at the mouth of pores provide sites where gaseous $\mathrm{H}_{2}$ can dissociate into atomic $\mathrm{H}$. The single $\mathrm{H}$ atoms can spill over onto the silicon support, then bond-hop by surface diffusion to fill the dangling bonds along the interior surfaces of the electrochemically-etched pores. This charging process requires less than 8 atmospheres of pressure $(8 \times 101 \mathrm{kPa})$. The discharge is accomplished by adding heat, with the release temperature determined by the action of the catalyst. Platinum-group catalysts were found to lower the energy barrier so effectively that a less-capable catalyst is needed. Transition metals such as iron and nickel offer an attractive alternative, and fortuitously, they are abundant in regolith.

Regolith is replete with silicon. Two methods are available for extracting purified silicon, and either may be used to produce the feedstock for making npSi for solid-state hydrogen storage. Both methods also produce purified iron. The first method is ball milling, wherein a rotating drum operating in a gravity field and containing spheres of dense, hardened material comminutes (fractures) the materials placed inside. This simple method has been demonstrated to disaggregate fusions of silicon and metal into their constituent elements, resulting in silicon with a purity greater than $99.999 \%{ }^{6}$. The silicon can be separated by sifting and settling in a gravity field and a gaseous environment, a process which can also segregate iron and nickel. Pure iron may be derived from the same ball-milling method, said iron having been electromagnetically harvested from the regolith as free iron, possibly embedded in metal agglomerates, using methods developed by the author ${ }^{10,11}$. Should elemental silicon be rare in a given body of regolith the patented methods of isotope separation by the author ${ }^{7,8,9}$ provide a second path for the production of pure silicon. This latter method of producing silicon involves ionizing regolith, forming a beam of plasma, then isolating the elements by their charge-to-mass ratio using a transverse electric field. Because all elements are included in the beam, it is therefore possible to simultaneously extract multiple purified substances. The individual isotopic beams can be formed such that they deposit onto a moving substrate making it possible to form patterns and shapes ${ }^{13}$. These directional deposition and 3D printing feature will become important later, as will the capability to produce pure titanium from the same beam. When asteroidal material is available, pure carbon can also be isolated.

Silicon is electrochemically etched to form the npSi matrix upon which the catalyst is deposited. Two methods exist to synthesize npSi, one suited to particulate silicon such as that from the ball milling purification, and the other suitable to single crystal silicon which can be made from the isotope separator. Particles of silicon can be porosified using what has historically been called a "stain" etch (originally used to identify crystal defects ${ }^{12}$ ). A stain etch produces a very high surface area within a granular material which can be readily packaged into almost any shape of the hydrogen storage vessel. The process to make single crystal silicon is more involved, but offers higher yield, and has the considerable added benefit of being directly suited to the fabrication of photovoltaic solar panels. Figure 2 outlines the steps involved where the dust roaster extracts oxygen gas ${ }^{27}$ (useful for life support and as propellant oxidizer) but also prepares the sub-oxide minerals for the plasma-based isotope separation process. Edge-fed growth extrudes thin slabs of crystal from a crucible. The extrusion has been started with a seed crystal, similar to how sourdough bread cultures are carried forward in time. For the purposes of this study, a singulated slab of single crystal silicon is sufficient for the electrochemical etch to form npSi.

The recipe for $\mathrm{npSi}$ is to pass $0.1 \mathrm{~A} / \mathrm{cm}^{2}$ through a solution of hydrofluoric (HF) acid with a surface tension-lowering alcohol such as ethanol or methanol. Discovered in the 1950s at Bell Labs this process produces a regular array of extremely tiny pores having an extremely high aspect ratio. No patterning is required, so making the silicon porous at the nano-scale can be done with simple equipment. The challenge with lunar operations is providing fluorine required for the etch, as lunar regolith has a vanishingly small concentration of this highlyreactive atom. The process for recycling and reconstituting spent HF is vital, and treated in

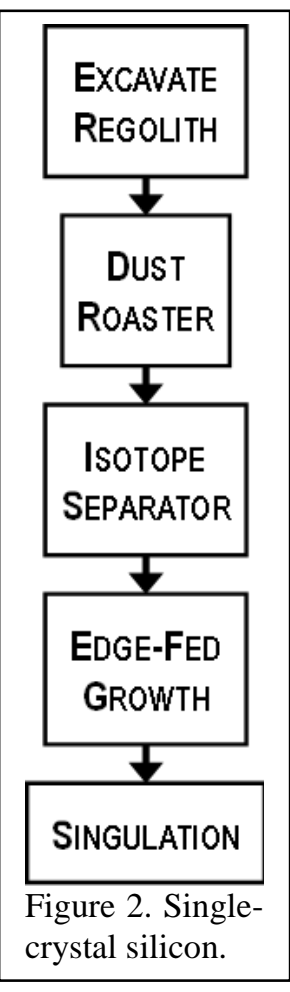
detail below.

American Institute of Aeronautics and Astronautics 
The result is a catalytically-modified hydrogen storage media which can be charged at 8 bar (the pressure of a racing bicycle tire) and discharge at temperatures below $120^{\circ} \mathrm{C}$. It must be packaged in a suitable vessel which includes means by which the temperature can be distributed throughout the interior. The traditional approach is a heated working fluid flowing through a network of pipes similar to how blood flows through the body. Another approach is to provide the heat via infrared (IR) heating which has the advantage of passing through de-hydrogenated npSi but being absorbed by $\mathrm{Si}-\mathrm{H}$ bonds, making it more selective, and requiring less volume and mass, as explained below.

Applying iron or nickel as a catalyst can be accomplished with wet chemistry but also from carbonyl vapors using waste $\mathrm{CO}_{2}$ from human habitats. The hydrofluoric acid needed can possibly be made from halogens in asteroids but these are very dilute, so the HF will likely need to be brought from earth. Byproducts of the synthesis is hydrofluorosilicic acid $\left(\mathrm{H}_{2} \mathrm{SiF}_{6}\right)$ which decomposes back to $\mathrm{HF}$ and $\mathrm{SiO}_{2}$. Thus with relatively simple but robust apparatuses these reagents can be recycled indefinitely (barring leaks). Together these methods create the media for solid-state hydrogen storage.

\section{Reconstituting Hydrofluoric Acid (HF)}

$\mathrm{F}$ luorosilicic acid (FSA) is the byproduct of forming npSi. FSA is an abundant and toxic industrial waste on earth, and over the last 50 years several methods for its recovery have been proposed and patented ${ }^{16,17,18,25}$. Others have sought to recover the waste silica for use as an abrasive or as a sorbent ${ }^{20}$. Silica is the main constitutent of white sand, and is really just granulated quartz. The novel process proposed herein recycles FSA back to HF needed for npSi synthesis, and also produces pure silica which can be used for portals, glassware, or as part of the IR lamps mentioned above and detailed in a subsequent section.

Electrochemically etching silicon in an HF acid mix produces npSi and FSA and releases hydrogen gas. The process disclosed here for the first time uses thermal decomposition only (existing processes require sulfuric acid as a reagent) with the only effluent being solid silica $\left(\mathrm{SiO}_{2}\right)$ and also hydrogen gas $\left(\mathrm{H}_{2}\right)$ which can be stored in npSi vessels and sold as an item of commerce as depicted in Fig. 1. Water is needed as an input to balance the outflow of oxygen and hydrogen, so that the overall "black box" reaction is:

$$
\mathrm{Si}+\mathrm{H}_{2} \mathrm{O} \rightarrow n p \mathrm{Si}+2 \mathrm{H}_{2}+\mathrm{SiO}_{2}
$$

The residual after npSi synthesis is a mixture of HF, alcohol, water, and FSA. FSA has a boiling point of $108{ }^{\circ} \mathrm{C}$, and that of methanol is $65{ }^{\circ} \mathrm{C}$. Thus, with a two stage fractional distillation these can be vaporized and then recondensed to reconstitute the HF+alcohol etch mix. At the BP of FSA water will also evaporate, and this leaves the silica bone dry and ready for removal. Each step of the process is conducted in batches although there is a closed loop for the fluorine-containing species. The thermal composition equation of interest is summarized by:

$$
\mathrm{H}_{2} \mathrm{SiF}_{6}+\mathrm{H}_{2} \mathrm{O} \stackrel{\text { HEAT }}{\Longrightarrow} 2 \mathrm{HF}_{(v)}+\mathrm{SiF}_{4(g)}+\mathrm{SiO}_{2(s)}
$$

A condensation apparatus common to the vapor and gas streams from both stages of the fractional distillation returns them to a plenum above the mixing reservoir. At a temperature of $10^{\circ} \mathrm{C}$ the methanol, $\mathrm{HF}$, and water will be in liquid form. Only the $\mathrm{SiF}_{4}$ will remain as a gas, thus a pump sited at the top of the plenum siphons it off and returns it to the first stage of the distillation where a sparger causes it to bubble through the solution there, where it becomes reabsorbed as new FSA. The apparatus design for this process is depicted in Figure 3 where the constituents of gas/vapor streams are listed in fonts indicative of their relative amounts. The precise temperatures used will affect the equilibrium concentrations and rates of the various reactions mentioned and this may require experimentation on the moon to determine the optimal setpoints. Ports for the introduction of water and silicon, and for the removal of hydrogen and silica, per equation (1) are shown as white arrows. Note that gravity is required making reconstitution of fluorine in this manner suitable only for planetary bodies.

American Institute of Aeronautics and Astronautics 


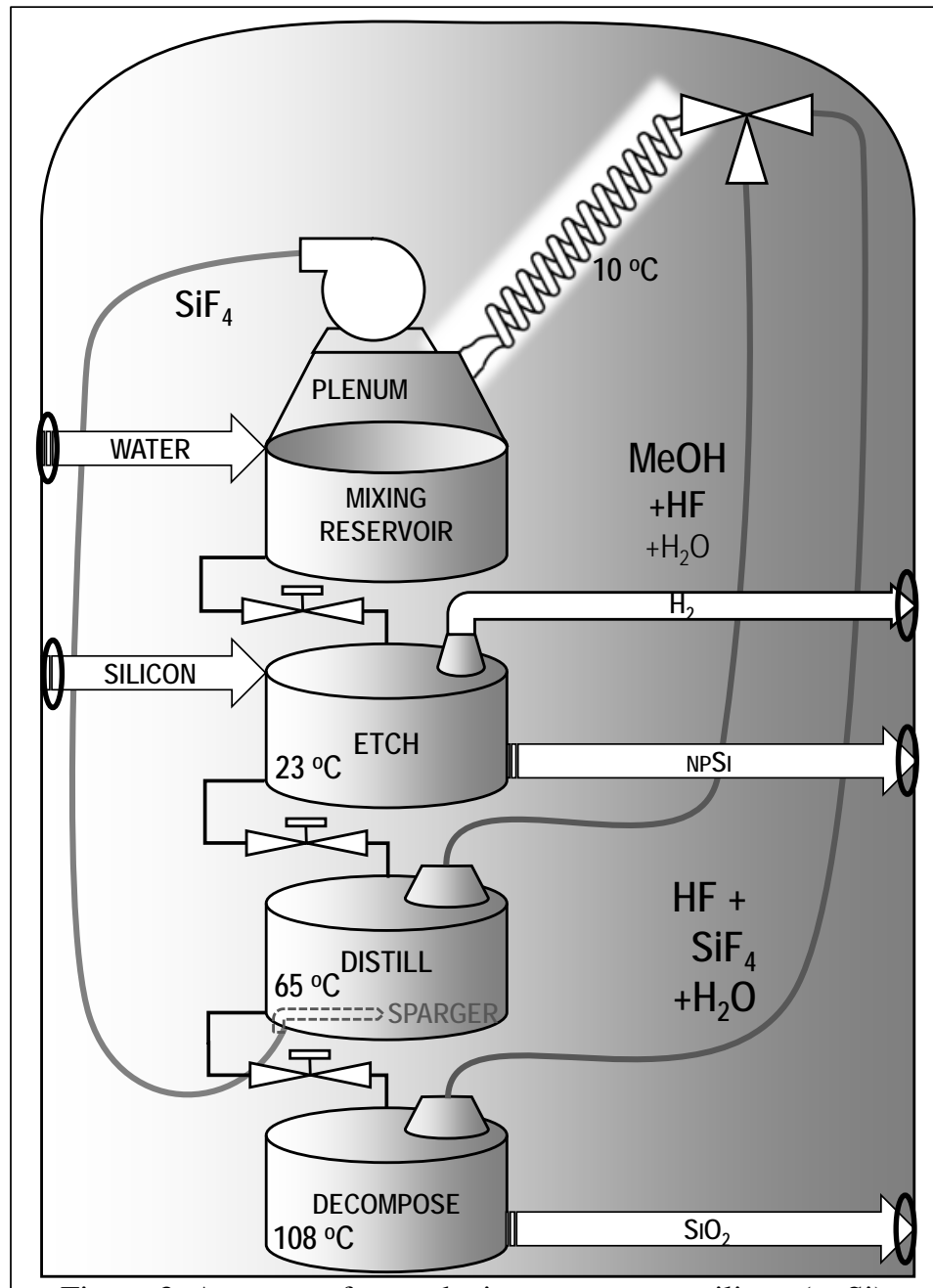

Figure 3. Apparatus for producing nanoporous silicon (npSi).

vapor deposition using hydrogen-based gases containing Si and C. A now-expired patent by the author ${ }^{14}$ has been applied to ISRU power transistors which can be fabbed in orbiting or planetary habitats using a process which requires no critical alignment steps (Figure 4). Circuits made from discrete devices, or low device count integrated circuits can be used for data processing, automated controls, and power management of both the process to build solid state hydrogen storage systems, but also the internal operation of those systems themselves.

\section{IR Emitters}

Infrared (IR) emitters are simple in concept, requiring an isolated wire to be heated by the passage of current until it glows. Depending on the power applied and the geometry and materials of the wire the temperature can be adjusted and thereby the blackbody radiation peak wavelength through the Wien's displacement law. A novel device configuration, designed to be compatible with ISRU materials and methods is introduced here for the first time, shown in figures 5 and 6.

\section{Electronics}

Silicon is a well-established semiconductor from which to make integrated circuits, solar panels, and now solid-state hydrogen storage media. Yet, computer chip fabs require an extensive array of chemical reagents, making it infeasible to transplant traditional methods to the moon. Another approach is to use silicon carbide ( $\mathrm{SiC}$ ), a semiconductor in many ways better suited to space. $\mathrm{SiC}$ trasnsitors and devices can withstand much higher temperatures and higher power densities than those made in silicon. Complexity cannot be as great because $\mathrm{SiC}$ crystals are difficult to make as defect-free, however individual devices are practical. To be an all-ISRU process requires the carbon to come from space, and that implies C-class or carbonaceous chondrite type asteroids. With carbon separated chemically, or with the isotope separator described above, it is possible to form $\mathrm{SiC}$ crystals via chemical

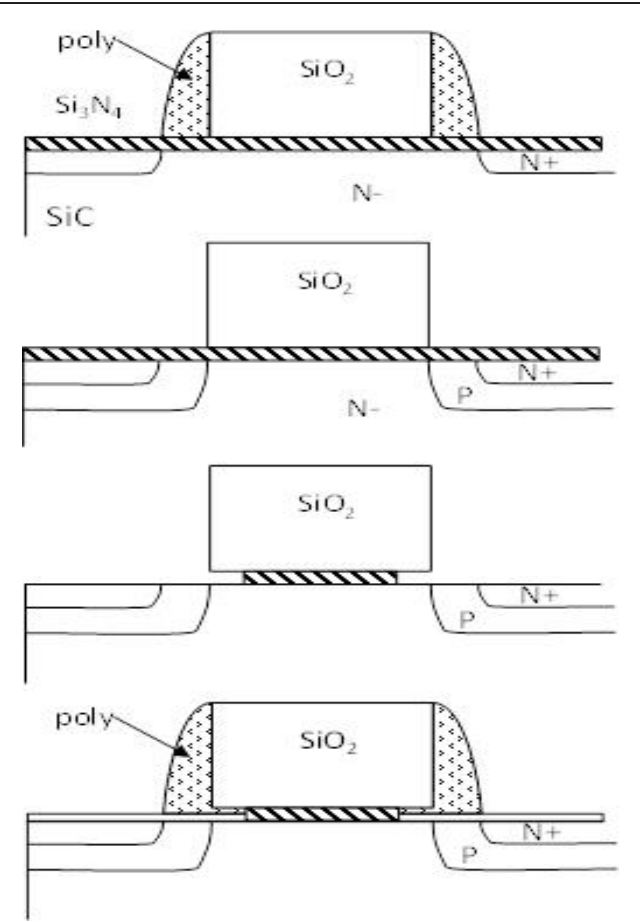

Figure 4. Cross section of key process steps for fabricating power transistors in $\mathrm{SiC}^{15}$. 


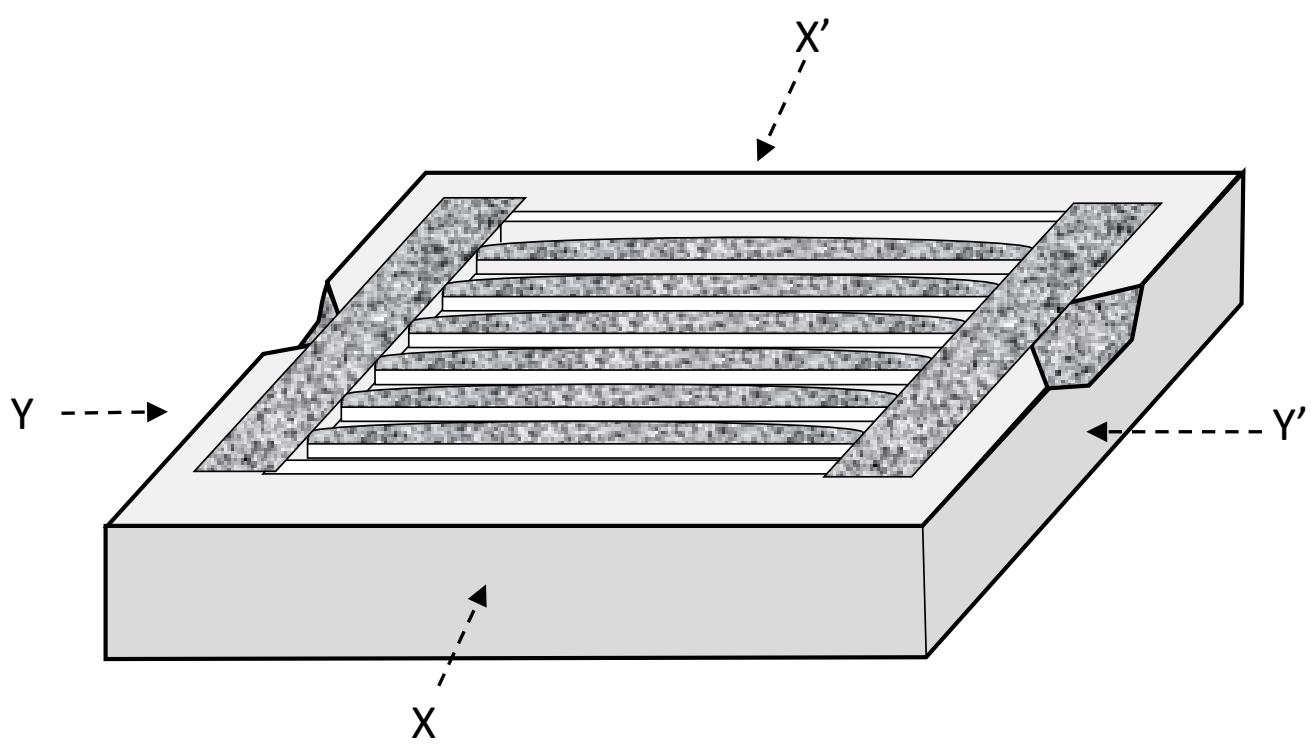

Figure 5. Perspective view of IR heater element (bonded quartz cap vacuum seal not shown) fabricated by embossing plus directional titanium deposition (textured areas). Arrows indicate cross sections in Fig. 6.
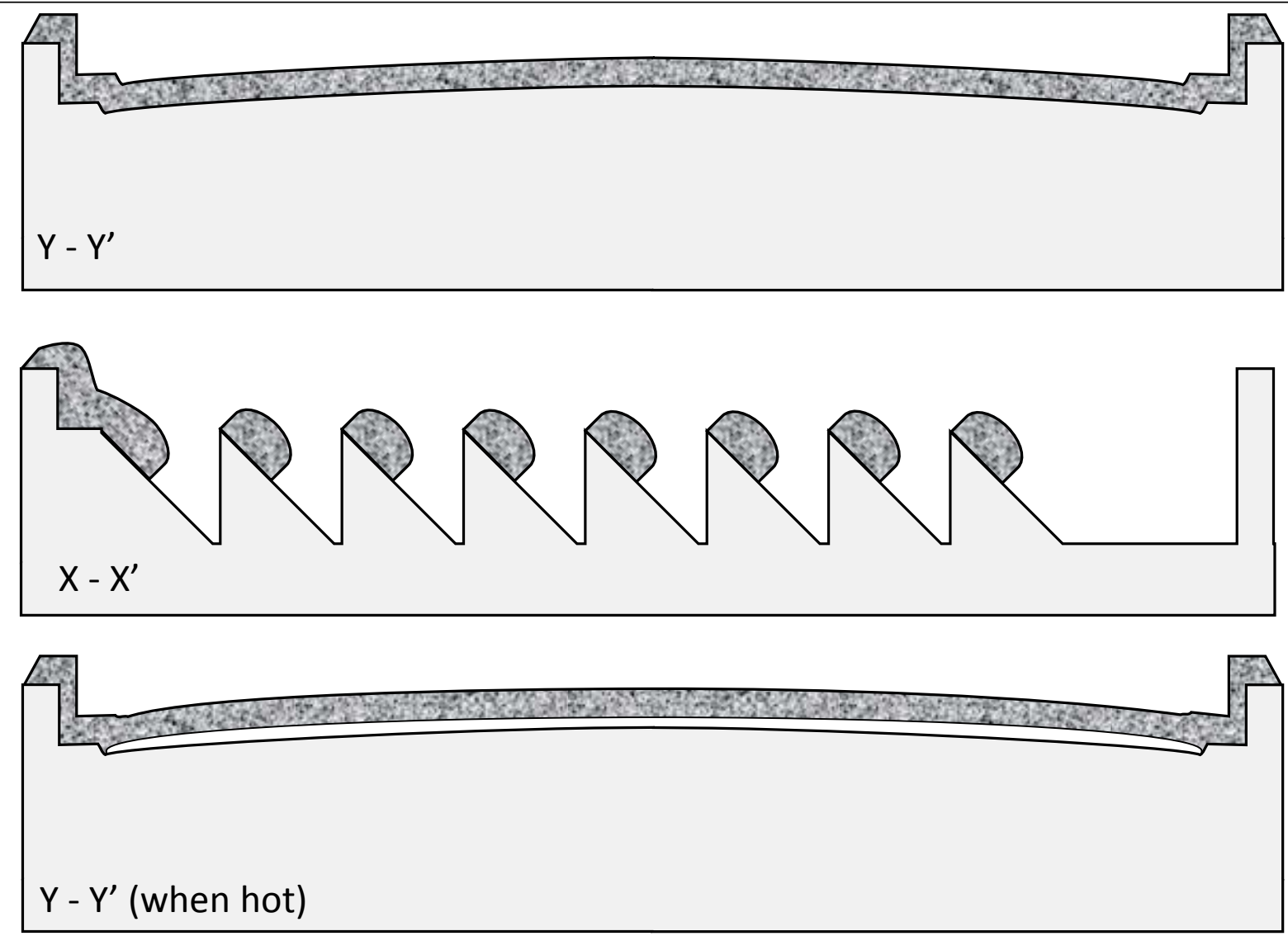

Figure 6. Cross section IR heater element per directions in Fig. 5. Bottom figure shows gap when filament is hot.

American Institute of Aeronautics and Astronautics 
Fabrication of IR emitters requires powdered silica, one of the effluents of the HF recovery process in section III. Using dies made from SiC, or other refractory material, the powder is pressed, or embossed, into the shape shown in Fig. 5 shown using a combination of pressure, temperature, and time sufficient for the silica to flow, conform, and expel void spaces. This process is ideally conducted in vacuum, and can be powered by concentrated sunlight. With adequate temperature the silica fuses into quartz crystal which is transparent to the IR light needed for rapid desorption of hydrogen from the catalytically-modified npSi storage media. Using the isotope separation process described above a stream of titanium ions is directed towards the completed, and cooled, quartz die at an angle of approximately 45 degrees such that the ridges indicated in the $X-X^{\prime}$ cross section of Fig. 6 shadow a portion of the metal beam leaving individual fingers of Ti metal traversing the Y-Y' cross section. Larger bond pads of Ti are formed at either end of Y-Y' (with outlines defined by a shadow mask) which are in electrical and mechanical contact with the Ti fingers, the latter having a smaller cross section and hence higher resistance. When a current is passed between the bond pads the higher resistance fingers experience greater Joule heating, raising their temperature, and expanding the titanium metal. As shown in the botton of Fig. 6 the curved shape of the finger substrate facilitates its expansion when hot to rise above the quartz so that the hottest portion is no longer in intimate thermal contact such that the thermal energy of the finger is dissipated predominantly via electromagnetic radiation. With suitable design and current this radiation is in the IR range at a wavelength which passes through silicon but is absorbed by the $\mathrm{Si}-\mathrm{H}$ bond ${ }^{28}$.

Not shown in Figs. 5 and 6 is a quartz cap which is bonded to the substrate using pressure, heat, and optionally an electric field in a method known as electrostatic bonding. This method is widely used to seal micro-electromechanical systems (MEMS). In this way a vacuum is formed around the Ti fingers to eliminate the potential for oxidation, for hydridation, or for heat loss due to convection. Titanium metal extending under the quartz cap bond, shown as beveled edges in Fig. 5, provide for contact outside of the vacuum. Any gaps can be plugged by directional Ti deposition to ensure a tight seal. Arrays of such IR emitters are placed interior to the hydrogen storage vessel outer wall to facilitate rapid and complete desorption of hydrogen when demanded by the user. While the original dies may come from earth, the dies can be fabricated from $\mathrm{SiC}$ derived from asteroidal material. In this way the fabrication of IR emitters can be entirely produced by ISRU.

\section{Catalysts}

The definition of a catalyst is a substance which facilitates or accelerates a chemical process yet does not get changed in so doing. One well-known property of catalysts in the petrochemical industry is the ability of metal catalyst particles to separate the covalent bond of molecular hydrogen ("dihydrogen" or $\mathrm{H}_{2}$ ), making it available for reaction with hydrocarbon molecules. Platinum is often cited as the most versatile catalyst, used, for example, to convert hydrogen and nitrogen from the air into ammonia $\left(\mathrm{NH}_{3}\right)$ which is an important fertilizer for crops. As another example, nickel metal is used as a catalyst to hydrogenate (add hydrogen to) lipid molecules to make margarine (imitation butter). The electrochemical etch with an HF solution to make npSi leaves the interior silicon surfaces saturated with hydrogen atoms. If the as-synthesized $\mathrm{npSi}$ is heated to $377^{\circ} \mathrm{C}$ the individual atomic hydrogen atoms will combine into dihydrogen and dissociate from the surface as a gas. However at this temperature the now-vacant silicon bonds tend to reform, or combine, with their neighbors making them less accessible. By depositing a catalyst near the mouth of the pores in $\mathrm{npSi}$ this process can be facilitated by separating the $\mathrm{H}-\mathrm{H}$ bond of dihydrogen, which is a strong bond (hence hydrogen's great value as a fuel). The catalyst will dissociate the dihydrogen into atomic hydrogen which can then move about on the surface of the catalyst particle, and from there spillover onto the silicon surface. In this way the solid-state npSi can be re-charged from a gas.

Platinum group metals are so facile at hydrogen separation that they provide insufficient barrier against hydrogenated npSi spontaneously releasing $\mathrm{H}_{2}$ at room temperature. Therefore a "less good" catalyst is needed, such as transition metals. Commonly used transition metal catalysts include chromium, iron, and nickel, which have outer electrons in two shells. Being common in regolith, iron is an appealing choice for ISRU use with npSi. Table 1 compares bond dissociation energies for various two-atom hydrogen-metal combinations ${ }^{27}$.

\begin{tabular}{|l|l|l|l|l|l|l|}
\hline Single bond & H-H & Si-H & Ni-H & Fe-H & Pd-H & Pt-H \\
\hline $\begin{array}{l}\text { Bond energy } \\
\text { kJ/mol }\end{array}$ & 435 & 293 & 240 & 148 & 234 & 330 \\
\hline
\end{tabular}

It is known that clusters of catalyst atoms can coordinate to separate the $\mathrm{H}-\mathrm{H}$ bond at temperatures lower than those associated with the energies shown in Table 1 . Recent $a b$ initio studies of clusters of four tetrahedrally-arranged Pd atoms reduced $\mathrm{H}_{2}$ dissociation energy to $45 \mathrm{~kJ} / \mathrm{mol}$, a reduction of $81 \%$. For operation at or around room temperature 
this energy barrier is too low for a shirtsleeve environment. This work has not yet been extended to transition metal clusters, however the reduction is expected to be a lower percentage. With both $\mathrm{Ni}$ and $\mathrm{Fe}$ to work with it may be reasonable to expect that a suitable dissociation energy barrier can be established (approximately $1.0 \mathrm{eV}$ ) for discharge temperatures in the range of $100-120^{\circ} \mathrm{C}$.

\section{Hydrogen Storage System}

Containers for the storage system can be fabricated from aluminum because the maximum pressure for recharge is a modest 8 bar; the design for individual modules is shown in Figure 7. When hydrogen is separated via electrolysis it will be at a low pressure, yet the pumping requirements for recharge of this solid-state hydrogen storage system are quite modest - especially when compared to cryogenic storage. Vessels can be modular, and therefore assembled into storage systems of almost any size. Volumetric density and gravimetric density are both comparable to cryogenic storage at the system level, so there is no penalty to rocket mass or size when using this method to store hydrogen. While the IR emitters can boost the release rate, the maximum release rate from solid state storage is expected to be lower than with liquid hydrogen, thus ascent boosters may be the only propulsion application for which this method may not be optimal. For all other applications such as Hohman transfer, injection, ferrying, plus fuel cell operation to power electric motors and provide drinking water, solid state hydrogen storage is an excellent and convenient choice.
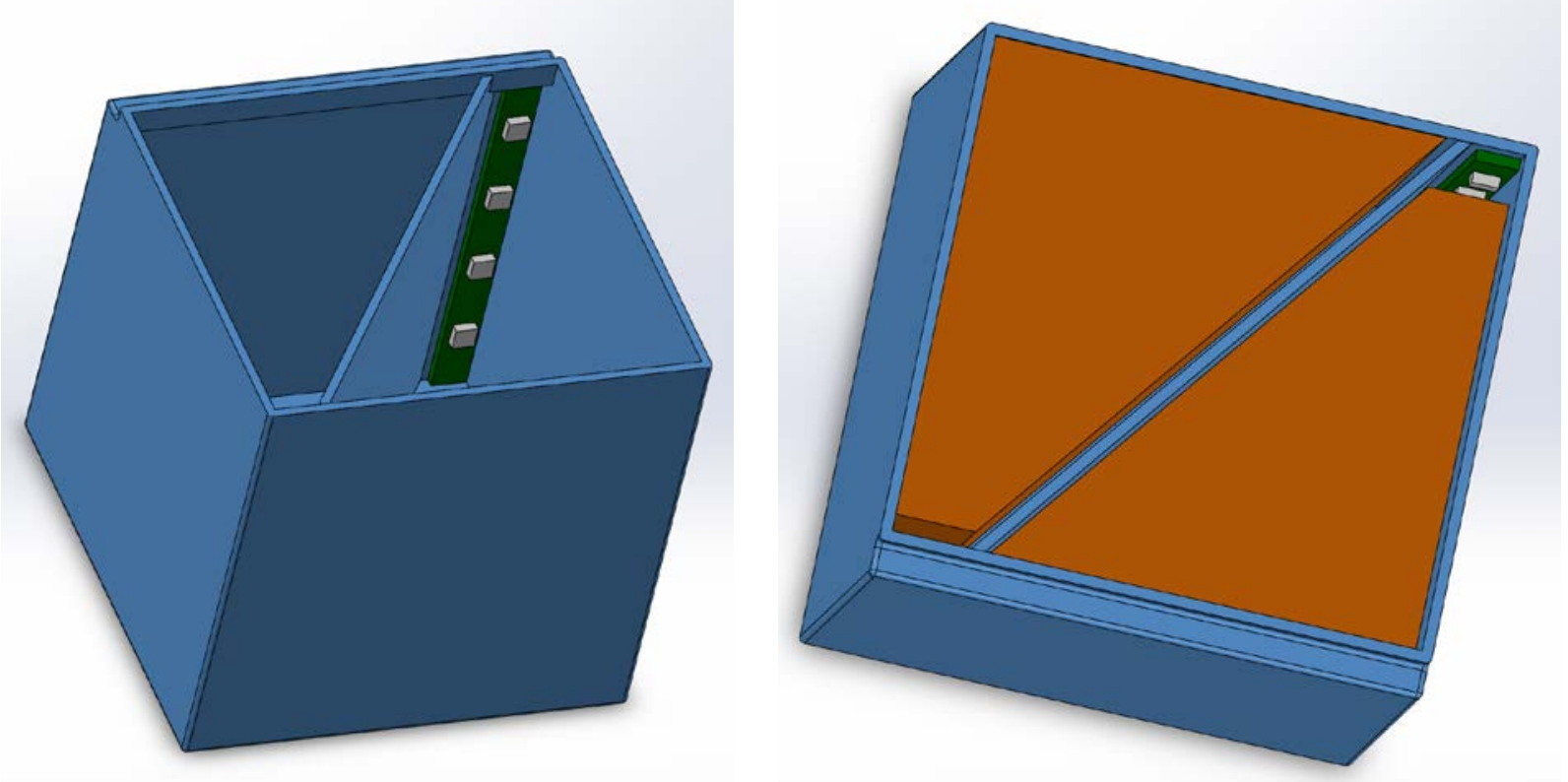

Figure 7. Modular storage showing internal IR emitters (left) and filled with (orange) granular npSi (right).

This paper ties together these various technology threads to integrate into a comprehensive hydrogen storage system which can be entirely produced from resources available in space, including control electronics and IR emitters. At the same time, it solves the problems of cryogenic hydrogen storage, and enables lower-energy, lower-mass ISRU operations to extract and store hydrogen from permanently-shadowed lunar craters or the interior of dead comets. The complete hydrogen storage system using these technologies is depicted in Figure 8. Note that any form factor, limited to the resolution of the individual modules of Fig. 7, can be built, so as to maximize interior volumes of habitats and spacecraft. These are important capabilities in the pathway to sustained operations in space, without the need to earthlaunch components, thereby reducing the energy and emissions associated with said launches. 


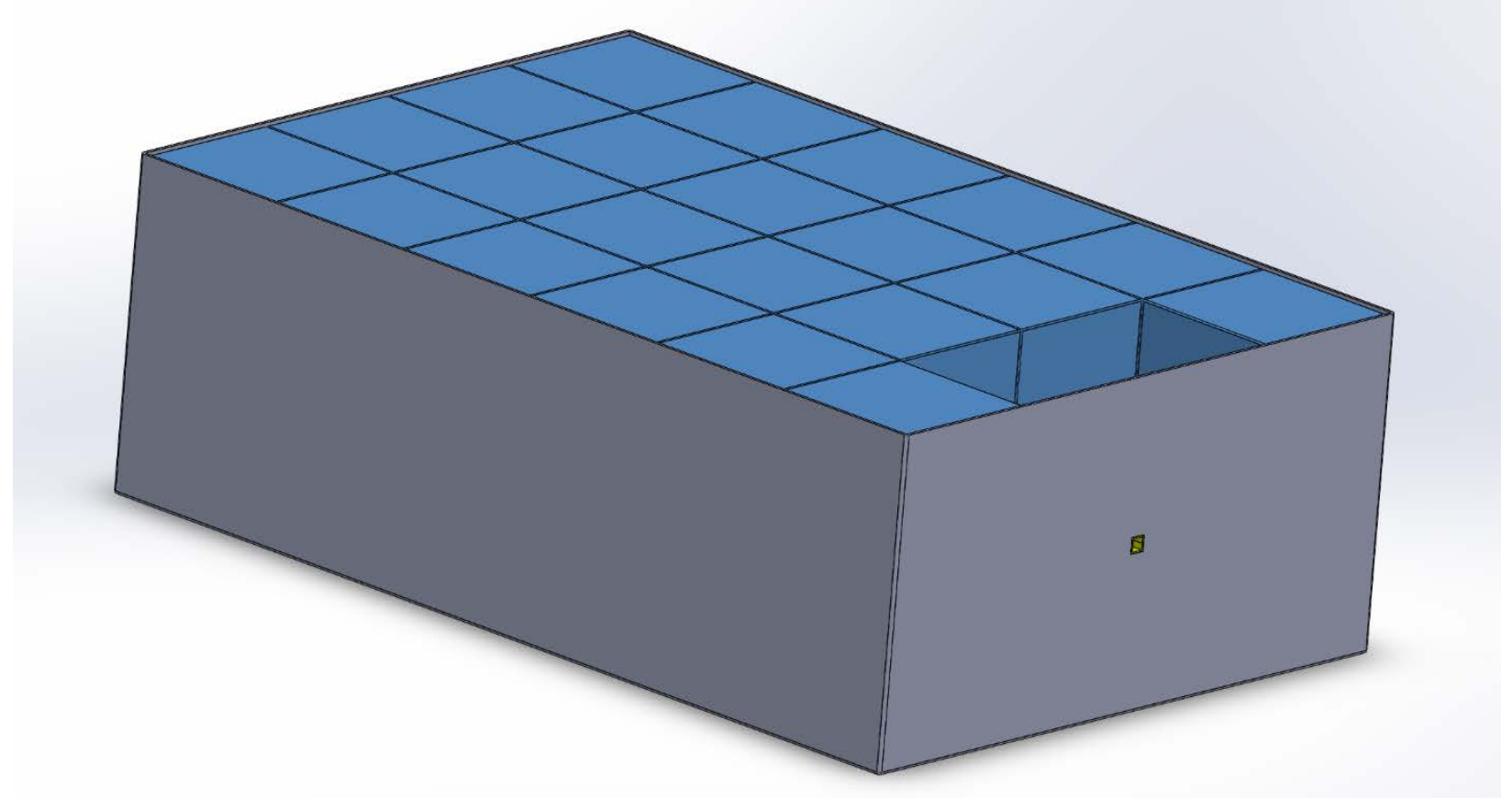

Figure 8. Complete hydrogen storage system fabricated $100 \%$ by ISRU.

\section{Discussion \& Next Steps}

While this work has identified the overall process for complete hydrogen storage systems using ISRU, and while some of the individual steps have been studied or demonstrated, there remain several which demand deeper study. The first is isotope separation using the patented methods described above. Needed is an end-to-end physico-chemical simulation so that an appropriate apparatus design can be derived, with throughput and yield predictions. Next is an ab initio analysis of the use of transition metal catalysts for mediating hydrogen storage on nanoporous silicon. The effort to complete this work using platinum group metals was a two year effort, however repeating this for other metals can now be completed more rapidly. Experimental confirmation of dissociation energy is important for all catalyst choices when deposited on the fractal topography of npSi.

This study isolates a single application but it is evident to the reader that there are many other products which can be created by the technologies described herein. Development of fundamental capabilities in purified raw materials, crystal formation, device manufacture and basic manufacturing capabilities is essential to establishing long-duration, high-population settlements on the moon, among the asteroids, or on Mars.

\section{References}

${ }^{1}$ Schubert, P., US 7,721,601, “HYDROGEN STORAGE TANK AND METHOD OF USING”, 2010.

${ }^{2}$ Schubert, P., US 7,833,428, "PROCESSES AND APPARATUS FOR PRODUCING POROUS MATERIALS," 2010.

${ }^{3}$ Schubert, P., US 8,518,856, "SOLID-STATE HYDROGEN STORAGE MEDIA AND CATALYTIC HYDROGEN RECHARGING THEREOF,” 2013.

${ }^{4}$ Schubert, P., US 8,673,811, "SYSTEM, METHODS AND MATERIALS FOR STORING AND RETRIEVING HYDROGEN,” 2013.

${ }^{5}$ Wilks. A.D., and Schubert, P., "Hydrogen Storage in Catalytically-Modified Porous Silicon,” NSF 1648748, Phase I STTR 1 Jan. 2017 to 31 Jan 2018.

${ }^{6}$ Zong, L, et. al., "Nanopurification of silicon from 84\% to $99.999 \%$ purity with a simple and scalable process," PNAS, 3 Nov 2015, v 112, no. 44, p 13473-13477. 
${ }^{7}$ Schubert, P., US 6,614,018, "PROCESS AND APARATUS FOR CONTINUOUS-FEED ALL-ISOTOPE SEPARATION IN MICROGRAVITY USING SOLAR POWER,” 2004

${ }^{8}$ Schubert, P., US 6.930,304, “ PROCESS AND APPARATUS FOR ISOTOPE SEPARATION IN A LOWGRAVITY ENVIRONMENT,” 2005.

${ }^{9}$ Schubert, P., US 7,833,428, “PROCESSES AND APPARATUS FOR PRODUCING POROUS MATERIALS,” 2010.

${ }^{10}$ Schubert, P., “A Novel Method for Element Beneficiation Applied to Solar Panel Production,” Space Exploration 2005, Albuquerque, NM.

${ }^{11}$ Schubert, P., Beatty, M., "Harvesting of Lunar Iron: Competitive Hands-on Learning,” Am. Soc. of Engineering Educators, Annual Conference, Pittsburgh, PA, June 2008.

${ }^{12}$ Vazsonyi., E., et. al, "Porous silicon formation by stain etching,” Thin Solid Films, V. 288, 1-2, 1 June 2001, p 295-302.

${ }^{13}$ Schubert, P., Moktar, K.A., Huang, X.E., Izam, M.I.M., "Metal Alloys for Additive Manufacturing plus Silicon and Oxygen from Regolith,” Int’l. Astro. Conf. 2016 poster IAC-16.A3.IP.27x32325, 28 Sept. 2016.

${ }^{14}$ Schubert, P., "Doubly-Self-Aligned Hole-Within-a-Hole Structure in Semiconductor Fabrication Involving a Double LOCOS Process Aligned with Sidewall Spacers," U.S. Patent number: 4,758,530.

${ }^{15}$ Schubert, P.J., "Silicon Carbide from Asteroids for Power Electronics," Int'l. Space Development Conf., San Juan, PR, 18-22 May 2016.

${ }^{16}$ Dahlke, T., Ruffiner, O., Cant, R., "Production of HF from $\mathrm{H}_{2} \mathrm{SiF}_{6}$," Procedia Engineering 138 (2016) p. 231-239.

${ }^{17}$ Cui-Hong Hou, Guang-Long Wang, and Bao-Lin Zhang, "Bubble Point Pressure of the Solutions of H2SiF6 + H2O and H2SiF6 + CO(NH2)2 + H2O from 323 K to 353 K,” J. Chem. Eng. Data, 2006, 51(3) p 864-866.

${ }^{18}$ Hartig, R.G., "Process for Recovery of HF and H2SiF6 From Gases Containing HF and SiF4," US 3,574,542, 1971.

${ }^{19}$ Holleman, A.F., Wiberg, E., Wiberg, N., Inorganic Chemistry, Academic Press 2001.

${ }^{20}$ Krysztafkiewicz, A, Swit, Z., Jesionowski, T., "Evaluation of Waste Silica Precipitated in the Process of Hydrofluoric Acid Production from Fluosilicic Acid,” Physicochem. Prob. Of Mineral Processing, 39 (2005) p 165-176.

${ }^{21}$ H. Lange, I.A. Schwirtlich, "Ribbon Growth on Substrate (RGS) - A new approach to high speed growth of silicon ribbons for photovoltaics,” July 1990 J. of Crystal Growth.

${ }^{22}$ Hahn G, et al. Review on ribbon silicon techniques for cost reduction in PV. In: Pro-ceedings of 4th World Conference on Photovoltaic Energy Conversion; 07-12 May, 2006,Waikoloa, HI (USA).

${ }^{23}$ Zawadzki, B., et al., "Method of Production of Anhydrous Hydrogen Fluoride," US 4062,930, 1977.

${ }^{24}$ Dreveton, A., "Manufacture of Aluminium Fluoride of High Density and Anhydrous Hydrofluoric Acid from Fluosilicic Acid,” Procedia Engineering 46 (2012) p 255-265.

${ }^{25}$ Parish, W.R., Kelley, J.C., Giovanetti, Al, Lutz, W.A., "Process for Producing Anhydrous HF from Fluosilicic Acid, US 3,758,674, 1973.

${ }^{26}$ Hofer, U, "Nonlinear optical investigation of the dynamics of hydrogen interaction with silicon surfaces," App. Phys. A, 63, p 533-547, 1996.

${ }^{27}$ Schubert, P., Meno, J., “Oxygen Extraction from Lunar Regolith via Free-Fall Induction Heating,” Intl Space Development Conf. Huntsville, AL, 19-22 May, 2011.US 7,935,176,

${ }^{27}$ Luo, Y.R., Comprehensive Handbook of Chemical Bond Energies, CRC Press, Boca Raton, 2007.

${ }^{28}$ Brodsky, M.H., Cardona, M., Cuomo, J.J., "Infrared and Raman spectra of the silicon-hydrogen bonds in amophous silicon prepared by glow discharge and sputtering,” Phys. Rev. B., v. 16, no. 8, 15 Oct. 1977.

American Institute of Aeronautics and Astronautics 